\title{
SULLE TRASFORMAZIONI CREMONIANE CHE POSSEGGONO PER CURVA DI PUNTI UNITI UNA SESTICA CON DIECI PUNTI DOPPI
}

\section{GIUSEPPE POMPILJ}

In una precedente memoria Sulla trasformazioni Cremoniane del piano che posseggono una curva di punti uniti ${ }^{1}$ ho messo in evidenza che tra $\mathrm{i}$ tipi possibili di siffatte trasformazioni si presentano quelle che hanno per curva di punti uniti una sestica con 10 punti doppi; per provare l'effettiva esistenza di tali trasformazioni ne ho dato un esempio (num. 21) che però è risultato essere insussistente perchè, come ha recentemente osservato $\mathrm{A}$. B. Coble, ${ }^{2}$ la trasformazione che si ottiene non è altro che l'identità. Perciò do quì un altro esempio.

La generica sestica razionale, con dieci punti doppi $P_{1}, P_{2}, \cdots, P_{7}$, $A, B, C$ di cui gli ultimi tre nei vertici del triangolo fondamentale $A(0,1,0), B(1,0,0), C(0,0,1)$, ha equazione parametrica:

$$
\begin{aligned}
& x=t\left(t-\alpha_{1}\right)\left(t-\alpha_{2}\right)\left(t-\gamma_{1}\right)\left(t-\gamma_{2}\right), \\
& y=t\left(t-\beta_{1}\right)\left(t-\beta_{2}\right)\left(t-\gamma_{3}\right)\left(t-\gamma_{4}\right), \\
& z=\left(t-\alpha_{1}\right)\left(t-\alpha_{2}\right)\left(t-\beta_{1}\right)\left(t-\beta_{2}\right)\left(t-\gamma_{5}\right)\left(t-\gamma_{6}\right) .
\end{aligned}
$$

Imponiamo a questa sestica di soddisfare alla seguente condizione: le due involuzioni individuate dalle coppie che cadono nei punti doppi $A, C$ e $B, C$ devono essere permutabili. Questo porta che sia $\alpha_{1} \alpha_{2}=-\beta_{1} \beta_{2}$ o meglio $\beta_{2}=-\alpha_{1} \alpha_{2} / \beta_{1}$; cioè si ha un solo legame tra $\mathrm{i}$ coefficienti $\mathrm{e}$ la condizione, come è naturale, risulta perfettamente simmetrica rispetto ai punti doppi $A$ e $B$. In questo caso l'equazione della nostra sestica $S$ diventa:

$$
\begin{aligned}
& x=t\left(t-\alpha_{1}\right)\left(t-\alpha_{2}\right)\left(t-\gamma_{1}\right)\left(t-\gamma_{2}\right), \\
& y=t\left(t-\beta_{1}\right)\left(t+\alpha_{1} \alpha_{2} / \beta_{1}\right)\left(t-\gamma_{3}\right)\left(t-\gamma_{4}\right), \\
& z=\left(t-\alpha_{1}\right)\left(t-\alpha_{2}\right)\left(t-\beta_{1}\right)\left(t+\alpha_{1} \alpha_{2} / \beta_{1}\right)\left(t-\gamma_{5}\right)\left(t-\gamma_{6}\right),
\end{aligned}
$$

ed un semplice esame di questa equazione parametrica ci assicura che nell'intorno dei tre punti doppi $A, B, C$ non esistono altri punti multipli di $S$.

Ma ancora con una sola condizione si può imporre che la sestica abbia:

${ }^{1}$ Rendiconti del Seminario Matematico della Università di Roma, (4), vol. 2 (1938).

2 This Bulletin, vol. 45 (1939). 
I. un punto triplo in luogo di tre punti doppi, o

II. un tacnodo, o

III. tre punti doppi su una retta, o

IV. sei punti doppi su una conica,

gli altri casi, come vedremo, non ci interessano perchè oltre che $P_{1}, \cdots, P_{7}$ fanno intervenire il punto doppio $C$ ovvero, per la simmetria, $A$ e $B$ contemporaneamente.

Sorge così il dubbio che la condizione imposta equivalga a qualcuna di quelle sopra elencate.

Il primo caso si esclude subito. Supponiamo infatti che la nostra sestica abbia un punto triplo $P$ in corrispondenza ai valori $\eta_{1}, \eta_{2}, \eta_{3}$ del parametro $t$; in tal caso le rette per $P$ segano ulteriomente $S$ secondo una $g_{3}^{1}$ che sul parametro $t$ è espressa da:

$$
\left(t-\alpha_{1}\right)\left(t-\alpha_{2}\right)\left(t-\delta_{1}\right)+\lambda\left(t-\beta_{1}\right)\left(t+\alpha_{1} \alpha_{2} / \beta_{1}\right)\left(t-\delta_{2}\right)=0 ;
$$

siccome poi questa $g_{3}^{1}$ deve contenere anche un gruppo del tipo $\left(0, \infty, \delta_{3}\right)$ segue che $\delta_{1}=-\delta_{2}(=\delta)$. Si osservi ora che le rette per $A$ e $B$ segano ulteriormente $S$ secondo due $g_{4}^{1}$ espresse, sul parametro $t$ dalle equazioni :

$$
\begin{aligned}
& t\left(t-\gamma_{1}\right)\left(t-\gamma_{2}\right)+\lambda\left(t-\beta_{1}\right)\left(t+\alpha_{1} \alpha_{2} / \beta_{1}\right)\left(t-\gamma_{5}\right)\left(t-\gamma_{6}\right)=0 \\
& t\left(t-\gamma_{3}\right)\left(t-\gamma_{4}\right)+\mu\left(t-\alpha_{1}\right)\left(t-\alpha_{2}\right)\left(t-\gamma_{5}\right)\left(t-\gamma_{6}\right)=0
\end{aligned}
$$

e queste due $g_{4}^{1}$ devono possedere rispettivamente due gruppi del tipo $\left(\delta, \eta_{1}, \eta_{2}, \eta_{3}\right),\left(-\delta, \eta_{1}, \eta_{2}, \eta_{3}\right)$ il che però porta un'ulteriore condizione sui coefficienti; possiamo quindi concludere che in generale la nostra sestica possiede 10 punti doppi e non un punto triplo e sette punti doppi.

Gli altri tre casi non sono riuscito ad escluderli. Preciserò perciò che cosa diventano nei vari casi le trasformazioni involutorie del quarto tipo di Bertini ${ }^{3}$ che hanno per fondamentali gli otto punti $\left(P_{1}, \cdots, P_{7}, A\right),\left(P_{1}, \cdots, P_{7}, B\right)$.

Se non si presenta nessuno dei casi del numero precedente, ovvero se la sestica $S$ ha un tacnodo si hanno due trasformazioni del $17^{\circ}$ ordine con i punti fondamentali: $\left(P_{1}^{6}, \cdots, P_{7}^{6}, A^{6}\right),\left(P_{1}^{6}, \cdots, P_{7}^{6}, B^{6}\right)$.

Se $P_{1}, P_{2}, P_{3}$ sono in linea retta si hanno due trasformazioni del $16^{\circ}$ ordine con $\mathrm{i}$ punti fondamentali: $\left(P_{1}^{5}, P_{2}^{5}, P_{3}^{5}, P_{4}^{6}, \cdots, P_{7}^{6}, A^{6}\right)$, $\left(P_{1}^{5}, P_{2}^{5}, P_{3}^{5}, P_{4}^{6}, \cdots, P_{7}^{6}, B^{6}\right)$.

Se $P_{1}, \cdots, P_{6}$ sono su una conica si hanno due trasformazioni del $13^{\circ}$ ordine con i punti fondamentali: $\left(P_{1}^{4}, \cdots, P_{6}^{4}, P_{7}^{6}, A^{6}\right)$, $\left(P_{1}^{4}, \cdots, P_{6}^{4}, P_{7}^{6}, B^{6}\right)$.

${ }^{3} \mathrm{~V}$. Snyder, The involutorial birational transformation of the plane, of order 17, American Journal of Mathematics, vol. 33 (1911). 
Cioè indicando con $b_{A}$ la trasformazione del quarto tipo di Bertini che ha i punti fondamentali $\left(P_{1}, \cdots, P_{7}, A\right)$ e con $b_{B}$ quella con $\mathrm{i}$ punti fondamentali $\left(P_{1}, \cdots, P_{7}, B\right)$ possiamo dire che le due trasformazioni $b_{A}$ e $b_{B}$ hanno rispettivamente i punti $A$ e $B$ come fondamentali e mutano i loro intorni in sestiche che passano doppiamente per $P_{1}, \cdots, P_{7}$ mentre hanno un punto triplo l'una in $A$ e l'altra in $B$.

Data la natura della sestica $S$ le due trasformazioni involutorie $b_{A}$ e $b_{B}$ sono permutabili su essa mentre, come dimostreremo, non lo sono sul piano; se infatti fosse $b_{A} b_{B}=b_{B} b_{A}$ siccome $B$ è unito per $b_{A}$ mentre è mutato da $b_{B}$ in una sestica irriducibile $D_{6}\left(P_{1}^{2}, \cdots, P_{7}^{2}, B^{3}\right)$ seguirebbe che questa sestica $D_{6}$ sarebbe unita per $b_{A}$ in contraddizione con il lemma VI (num. 10) della mia memoria precedentemente citata. Da ciò segue che la trasformazione $Q=b_{A} b_{B} b_{A} b_{B}$ non è identica ed ha la sestica $S$ come luogo di punti uniti.

Questa trasformazione $Q$ non ha però tutti e dieci i punti doppi di $S$ come fondamentali; per averne una che soddisfi anche a questo requisito, basta prendere una trasformazione cremoniana $T$ la quale oltre che mutare in sè la sestica abbia per fondamentali quei punti doppi di $S$ che non lo sono per $Q$, e quindi costruire la: $R=T^{-1} Q T Q^{-1}$.

R. UNIVERSITÀ, ROME, ITALY 